\title{
A Presentation of the Methodology Used in an Exploratory Study for the Purpose of Generating Hypotheses Regarding the Relationships Between An Individual's Daily Life and Aesthetics
}

\section{Catherine Ballard}

\author{
Introduction
}

When I first began the process of developing my dissertation topic I was sure of one thing; I was interested in the notion of aesthetics. I quickly became aware that, as Vincent Lanier stated: "Questions of aesthetics are by no means simple and obvious, indeed they are in their present substance, complex and tenuous as well as inadequately developed" (1977, p. 132). In particular, I perceived a lack of inquiry addressing relationships between aesthetics and daily life activities. Also many discussions of aesthetics appeared to be based on philosophical assumptions and definitions of aesthetics that were not fully grounded in data derived from 'everyday' experience. Initially I was motivated by Joseph Kupfer's (1983) statement, "Our understanding of the aesthetic is completed through our inquiry into the everyday, and our grasp of the problematic nature of daily life is deepened by our aesthetic approach" (p. 3). This proposal for an investigation of human interests and activities which comprise daily living was further supported by anthropologist Toni Fratto (1978). He suggested that an examination of categories from daily life, such as 'work,' could contribute to an understanding of aesthetics.

As the direction of my research became more clearly defined, it also became apparent that an exploratory study would be most appropriate. An important facet of this process involved the identification of a methodology that could provide a guiding structure with inherent flexibility and capable of coping with a potentially complex inquiry. I consider myself fortunate to have been working in a department where others have had similar interests and concerns. Consequently I was provided with the guidance and support necessary to complete this type of research. I would like to take this opportunity to present some background and a description of the methodology used in my study.

\section{Orientation and Purpose of the Study}

The spectrum of aesthetic inquiry and discussion ranges from the philosophical or speculative to empirically based research generally falling under the label of 'experimental aesthetics.' While both of these approaches may provide some insight into this complex area of study, my research is based on an orientation that is situated between these two 'extremes.' By 
adopting a grounded theory approach (Glaser and Strauss, 1967) and adapting ethnographic field work techniques, this descriptive and exploratory study has focused on collecting qualitative data about an individual's activities, interests, and personal background. These data are being examined for emergent categories of valuing patterns. Based upon the relationships between these categories, hypotheses regarding aesthetic valuing will be generated. An underlying purpose of this type of study is aimed at generating questions for future research that have been grounded in data. This approach to research assumes that hypotheses generating studies should be done prior to hypotheses testing studies. This style of research attempts to determine what some of these relevant research questions might be.

\section{Design Features of the Study}

There are five features in the design of this study. These are: 1) the grounded theory approach; 2) emphasis on an emic perspective; 3 ) use of ethnographic field work techniques; 4) the Jones Non-Quantitative Data Analysis Method which includes a Visually Weighted Free Keyword Indexing System; and 5) resulting techniques for generating hypotheses from nonquantitative data.

Glaser and Strauss's (1967) grounded theory approach is the guiding structure for the design of this study. This theory stresses the systematic generation of theories from data rather than verifying previous theories by "logical deduction from a priori assumptions" (p. 3). It's purpose is to develop theories which are firmly grounded on rich data instead of other predetermined theories. While Glaser and Strauss's theory is useful in placing one study within a long-term theory-generation process, specifics regarding data collection and analysis is lacking. The compatibility between the grounded theory approach and the general ethnographic emphasis on a dialectical process and holistic perspective (Agar, 1980) indicates a source of other methods and field work techniques that can be used to supplement the grounded theory approach.

These frameworks indicate a bias toward descriptive research and presuppose that the data collected will be primarily qualitative, that the result of the study will be interpretive in nature, and that the reliability and validity of the study should be viewed in terms of ethnographic research (LeCompte \& Goetz, 1982). The adoption of a grounded theory approach also indicates that no conclusive statement will be produced. As a contribution to "emerging theory" (Glaser \& Strauss, 1967) the present study will generate only a few, between two and five, of the many possible hypotheses for future hypothesis testing and theory generation. In this approach, the researcher becomes a primary research tool and his/her knowledge, sensitivity, awareness, and breadth of perception are limitations of this type of study (Yuan, 1986, pp. 9-11). The use of the Jones Visually Weighted Free Keyword Indexing System (1988), and its resulting hypothesis-generation technique determines that completely different results could be obtained by different researchers or

Working Papers in Art Education 1989 - 1990 
by the same researcher focused on different aspects of the data. However, all hypotheses may be checked against original observational data in context.

These five design features were incorporated into a four stage process. These were: 1 ) the planning stage; 2 ) the orientation stage; 3 ) the exploratory stage; and 4) the hypothesis-generation stage.

\section{Four Stage Process}

\section{The Planning Stage}

During the planning stage, efforts were focused on formulating the research problem and acquiring conceptual tools. This was accomplished primarily through a review of literature. A second major task completed during this stage was the identification and initial contact with my primary informant, Katherine. As a case study of a single individual, it was important to determine Katherine's willingness and commitment to participate in the project. I am pleased-to report that the precautionary 'back-up planning' I did, in the event that some unforeseen circumstance prevented Katherine from continuing with the project, was not needed. Over the course of a year's discussions, I felt that Katherine maintained a cooperative attitude towards the project.

\section{Selecting an Informant}

I first met Katherine through a mutual friend about one and a half years prior to our involvement with this project. During that time my interaction with her was limited to relatively brief conversations at New Zone openings. Both Katherine and my friend were members of this local cooperative art gallery. Through these brief encounters as well as social conversations with other New Zone members, I became aware of some of Katherine's background, experience, current interests and activities.

The process that led to my approaching Katherine about this project, involved a series of considerations. These emerged from questions such as, Did I want to work with a group or an individual? What were some of the 'essential' qualities and characteristics of both the individual and our circumstances? First I decided to work with a female from my own culture. Because I was interested in exploring aesthetics through a contextual examination of 'daily life,' it was not generally important whether or not this person was an artist or possessed any particular 'expertise.' I say 'generally' because I did have some concerns about working with an artist. I initially planned to de-emphasize the category of art, and focus more on 'everyday' activities. Consequently I was concerned that if this person was an artist, the role of their artistic involvement in relation to other aspects of their lives might emerge as an influential consideration. It also seemed preferable that she be someone with whom I was already acquainted, but was not a close friend or colleague. This was important for two reasons. First, given the time frame of the study and to aid in the development of rapport, it was considered

Working Papers in Art Education 1989 - 1990 
beneficial if we could begin the project with some pre-established sense of familiarity and comfort with one another. At the same time, because shared assumptions are often taken-for-granted in established friendships, it was important that we should not know each other too well. Similarly, because I planned to work with someone from my own culture, it was also important that she have some areas of interest distinctly different from my own. As these considerations became clear, Katherine emerged as someone I should talk with about the project. She is a Eugene based middle school Spanish teacher, political activist and artist, and a self-identified feminist and work-aholic. Katherine possessed all the characteristics which I required. Following a discussion of my intentions, Katherine enthusiastically expressed her interest in being my 'informant' for the study.

During this initial conversation an important characteristic of the study was emphasized. This related to the kind of working relationship I hoped to promote during the course of the study. I preferred to think of the study as a 'collaborative project' rather than thinking in terms of 'researcher informant relationships' and 'doing a study.' An ethical consideration and acknowledged potential limitation of this study, relates to adaptations made during the project to insure that Katherine's needs and personal sense of benefit from our involvement were met.

\section{The Orientation Stage}

The orientation stage was used to prepare for field work through more detailed, although still preliminary, discussions with Katherine. This stage further developed our rapport and assisted in understanding the general context of Katherine's activities and background, This was done to facilitate decision making about appropriate field work procedures and methods. During this time, my literature review continued. Increasing familiarity with Katherine's interests and the emergence of additional needs regarding methodological techniques required extension of the literature review.

\section{The Exploratory Stage}

Ethnographic field work techniques were used during the explanatory stage to gather and record contextual data regarding Katherine's activities, interests, and background, These techniques included: 1) unstructured informal conversations and taped in-depth interviews; 2) observation and participation; 3) journal-keeping; 4) photographic records of Katherine's work; and 5) a review of some personal artifacts, including printed and video records. Related to these techniques, and an important facet of this approach to research, is the recognition of the researcher as the primary tool in gathering and recording data. As previously indicated, sensitivity and selfawareness were key research instruments and acknowledged limitations of the study. 


\section{The Hypothesis Generation Stage}

Preliminary analysis of the data was necessary to provide some focus and direction in the exploratory stage. The hypothesis generation stage was based on a detailed and in-depth examination of the data. The 'Jones System' provided the analysis techniques used in this stage of the study. This system involved three related processes that were designed to categorize nonquantitative data through free key word indexing and visual sorting. Its aim is to help researchers, faced with a multitude of data in different forms and embedded in context, to derive categories from the information gathered.

The first of these processes involved transferring the data onto index cards and devising a related set of color codes and symbols. This included the use of different colors of index cards to indicate different types of data sources -- such as interview or observational data, etc. In order to transfer the data, I needed to identify some preliminary general categories and develop related visual symbols. These were based on recurrent topics or themes that seemed present in the data, and included things such as: time, teaching, work, self, relationships, thinking processes, artwork, and political activities, among others. A useful feature of the Jones system is that these categories need not be uniform or mutually exclusive. One data card could be linked to more than one category and contain several symbols. The size of the symbol, small, medium, or large, was also used to indicate degree or type of connection.

Another important feature of the Jones system is that crossreferencing among categories, levels of categories, and different subject areas is possible, rapid, and multidimensional due to our ability to assimilate visual information. It is thus possible for the cards to reflect considerable complexity and allow for greater flexibility in the interpretation of data. The use of visual symbols and color codes made it possible to sort the data using a variety of sorting schema, thus facilitating the emergence of additional categories, patterns and relationships. This indicates the second related process. One such schema involved looking for reoccurring clusters of symbols among the first level of categories. For example, in Katherine's case, the 'work' symbol was most frequently associated with the categories of relationships, self, thinking processes, and teaching. As the categories and their properties become more defined, the visual symbols were refined and new ones developed as needed. This in turn led to additional layers of interpretation. For instance, the category of 'work' was linked to areas such as decision making, commitment, responsibility, sense of self, and worldview.

As these processes involved 'partial' decontextualization and resorting of the data, each card was also indexed to include the original source and location of the data. This made it possible to trace various interpretations back to, and examine them in relation to the original data and their context. Additionally, the data were also only 'partially' decontextualized, because no patterns of relationships were pre-determined. The results of data analysis

Working Papers in Art Education 1989 - 1990 
were thus predictably less rigid than other data-analysis systems. This method of sorting and examining the data acknowledges and to some extent addresses problems with culturally conditioned information processing habits of Western-trained researchers. In summary, the resulting categories and properties of categories tend to be contextual, interrelated, and grounded on data (Jones, 1988).

The third related analysis process is begun after categories and properties of categories have been derived from the data, and involved the generation of hypotheses related to purposes of the study. The technique for doing so is based on an extension of the Jones System and the grounded theory approach. This phase of the research stresses the following: 1) that the categories and their properties are based directly on the data and may be traced back to original sources for review of analysis or further interpretation in relation to the generated hypotheses; 2 ) that the categories can be examined holistically in terms of interrelationships and networks; and 3) that relationships and networks deemed most relevant to the study's purposes of formulating hypotheses can be selected and examined further.

As I am still involved in this third process, I apologize for not being able to provide the reader with any hypotheses at this time. Given the exploratory nature of this study, 1 expect that even after the dissertation document is 'completed,' my examination of this data will not be finished. In closing I remind the reader that this particular case study ultimately needs to be considered in combination with other such studies, and in terms of longer term aesthetic research considerations. Its aim was not to arrive at any 'conclusions,' but rather to identify some future, potentially useful, research questions regarding aesthetics and daily life, thereby contributing to a grounded-theory of aesthetics.

\section{References}

Agar, M. (1980). The professional stranger. New York: Academic Press, Inc.

Fratto, T. (1978). Undefining art: Irrelevant categorization in the anthropology of aesthetics. Dialectical Anthropology, 3 (2), 129-138.

Glaser, B. G., and Strauss, A. L. (1967). The discovery of grounded theory: Strategies for qualitative research. New York: Aldine Publishing Co.

Jones, B. (1988). Unpublished lecture, University of Oregon, Eugene.

Kupfer, J. (1983). Experience as art: Aesthetics in everyday life. Albany, N.Y.: SUNY.

Lanier, V. (1976). Essays in art education: The development of one point of view. New York: MSS information Corp. 
LeCompte, M. D. and Goetz, J. P. (1982). Problems of reliability and validity in ethnographic research. Review of Educational Research, 52 (1), 31-60.

Wasson, R. F. (1983). The construction of an index for hypothesis generation concerning the art of Australian Aborigines in the process of culture change. Unpublished doctoral dissertation, University of Oregon, Eugene.

Yuan, J. (1986). An exploratory study for the purpose of generating hypotheses concerning the emic aesthetic valuing of a group of Taiwanese temple participants regarding temple art. Unpublished doctoral dissertation, University of Oregon, Eugene. 